\title{
Development of a PCR assay for the detection of nifH and nifD genes in indigenous photosynthetic bacteria.
}

\begin{abstract}
Molybdenum (Mo) nitrogenases consist of two components: dinitrogenase reductase (encoded by nifH) and the dinitrogenase or MoFe protein (encoded by nifDK). Nitrogenase enzyme of photosynthetic bacteria is responsible for hydrogen production. Therefore, primers were designed for the nitrogenase gene only. In this study, two primers (ND and NH) were designed after comparative genomic analysis of nifH and nifD gene sequences from public databases. The designed primers were used for the amplification of nifH and nifD genes to detect nitrogenase genes in photosynthetic bacteria. Initial detection was done using a monoplex Polymerase Chain Reactions (PCRs) followed by optimization of the PCR protocols. Subsequently, a duplex PCR was designed for amplification and detection of nifH and nifD genes in indigenous photosynthetic bacteria. Evaluation of the duplex PCR on six samples isolated from Palm Oil Mill Effluent (POME) showed that only four isolates contained both the nifH and nifD genes, indicating that these isolates were potential hydrogen-producing bacteria. PCR detection provides a rapid and efficient pre-identification of potential photosynthetic bacterial hydrogen producers.
\end{abstract}

Keyword: Biohydrogen; Nitrogenase; PCR detection; nifH; nifD; Photosynthetic bacteria. 